\title{
GRAPHICAL REPRESENTATION OF GRAIN AND HILLOCK ORIENTATIONS IN ANNEALED Al-1\%Si FILMS
}

\author{
D. GERTH and R. A. SCHWARZER \\ Institut für Metallkunde und Metallphysik der TU, Grosser Bruch 23, \\ D-38678 Clausthal-Zellerfeld, Germany
}

(Received 8 December, 1992)

\begin{abstract}
The grain-specific texture of $\mathrm{Al}-1 \% \mathrm{Si}$ films on oxidized silicon substrates was studied by transmission electron microscopy. Heat treatments at $400^{\circ} \mathrm{C}$ and $550^{\circ} \mathrm{C}$ gave rise to the sporadic growth of annealing hillocks. Crystal orientations of individual grains and hillocks were determined by means of on-line interpretation of Kikuchi patterns. Many hillock orientations differ from the sharp $\langle 111\rangle$ fibre texture of the layer. The hillocks are assumed to start growing in layer regions where grain orientations deviate from the main $\langle 111\rangle$ fibre texture. Local texture is represented graphically by orientation images using Miller indices, Euler angles, and Rodrigues vectors as orientation parameters.
\end{abstract}

KEY WORDS Individual grain orientations, orientational mapping, Rodrigues space, TEM, Kikuchi patterns, annealing hillocks, aluminium.

\section{INTRODUCTION}

During thermal treatment of thin aluminium films on silicon substrates, hillocks may grow on the surface of the films in order to relax compressive mechanical stress caused by the mismatch of thermal expansion between the metal layer and the substrate. The dependence of hillock growth on deposition and heating temperature, layer thickness, and stress was the subject of many investigations (Pennebaker, 1969; Lahiri, 1970; Bacconier et al., 1988; Chang et al., 1989; Kim, Hummel, 1990; Ericson et al., 1991). A review is given in (d'Heurle, 1989). Several types of hillocks have been reported: hillocks with crystal faces, rounded hillocks, hillocks on top of a single grain, and hillocks located on grain boundaries or triple point junctions. Single as well as polycrystalline hillocks were found.

In some investigations the growth process was studied in-situ in an electron microscope (Zimmermann, 1988; Smith et al., 1991; Gerth et al., 1992a). Hillocks tend to grow rapidly above a critical temperature which depends on the film thickness. During further annealing at higher temperatures the hillocks increase in size. They can also coalesce with the underlying grains, and polycrystalline hillocks may recrystallize. The changes of morphology and orientation of the hillocks and adjacent grains during the heating process is one reason for various shapes of hillocks found in some investigations after growth process.

Theoretical studies of hillock growth including computer simulation and comparison with stress relaxation mechanisms were made (Chaudhari, 1974; 
Jackson et al., 1982; Bacconier et al., 1988; Sanchez, Arzt, 1992). The main hypothesis is that hillocks grow on grain boundaries or triple point junctions, and that mass transport takes place by grain boundary diffusion (d'Heurle, 1989). The origin and the mechanisms of growth are not well understood yet. Obviously local processes in the region of individual hillocks have to be studied for describing the origin of the hillocks and the mechanisms of mass transport. One important aspect related to these questions is the crystal orientations of the hillock and the neighbouring grains. Because of the anisotropy of stress and the dependence of grain boundary mobility and grain boundary diffusion on the type of the grain boundary, the local misorientation is assumed to play an important role during hillock growth.

Here we present a transmission electron microscope (TEM) study of hillock growth. The crystal orientations of hillocks and grains were determined using the Kikuchi pattern method (Schwarzer, 1990). The findings are illustrated by orientation images of the grain structure.

\section{REPRESENTATION OF LOCAL TEXTURE BY ORIENTATION IMAGES}

\section{Description of an Orientation}

The crystal orientation is defined by the orientation of the crystal coordinate system $\{C\}$ with respect to a reference coordinate frame $\{S\}$ of the sample (Bunge, 1982). An orientation may be expressed by a rotation matrix $g$ which gives a mathematical description of the formalism to bring one coordinate system in correspondence with the other:

$$
\{C\}=\mathbf{g} \cdot\{S\}
$$

The sample reference frame $\{S\}$ is rotated into the crystal reference system $\{C\}$. In matrix notation it is:

$$
\left(\begin{array}{l}
c_{1} \\
c_{2} \\
c_{3}
\end{array}\right)=\left(\begin{array}{lll}
g_{11} & g_{12} & g_{13} \\
g_{11} & g_{12} & g_{13} \\
g_{11} & g_{12} & g_{13}
\end{array}\right) \cdot\left(\begin{array}{l}
s_{1} \\
s_{2} \\
s_{3}
\end{array}\right)
$$

The components of $\{S\}$ and $\{C\}$ are the three orthogonal basic vectors of the coordinate systems. The matrix $g$ can be expressed by using Euler angles, Miller indices, or rotational coordinates. It is possible to transform the different orientation parameters into each other by a comparison of the matrix elements. The formulas are given in (Hansen, 1978; Bunge, 1982). In case of cubic crystal symmetry 24 symmetrically equivalent descriptions $g^{e}$ of an orientation exist in a right-handed coordinate system. They are determined by multiplication of one orientation $g$ by the 24 symmetry elements, $g^{c}$, of the group 432 (Hansen, 1978):

$$
\mathbf{g}^{e}=\mathbf{g}^{c} \cdot \mathbf{g}
$$

If a sample symmetry is considered, further symmetrically equivalent orientations 
$\mathbf{g}^{e}$ are given by the relation:

$$
\mathbf{g}^{e}=\mathbf{g}^{c} \cdot \mathbf{g} \cdot \mathbf{g}^{s}
$$

where $g^{s}$ are the symmetry elements of the sample.

The Euler angles describe three rotations which transform the sample reference frame into the crystal frame. The coordinate system is first rotated around the $z$-axis, then around the new $x$-axis, and finally around the new $z$-axis. The three angles of rotation are $\phi_{1}, \Phi, \phi_{2}$. The rotation matrix $g\left(\phi_{1}, \Phi, \phi_{2}\right)$ is then given by (Hansen, 1978; Bunge, 1982):

$$
\mathbf{g}\left(\phi_{1} \Phi \phi_{2}\right)=\left(\begin{array}{cc}
\cos \phi_{1} \cos \phi_{2}-\sin \phi_{1} \sin \phi_{2} \cos \Phi \\
-\cos \phi_{1} \sin \phi_{2}-\sin \phi_{1} \cos \phi_{2} \cos \Phi \\
\sin \phi_{1} \sin \Phi \\
\sin \phi_{1} \cos \phi_{2}+\cos \phi_{1} \sin \phi_{2} \cos \Phi & \sin \phi_{2}-\sin \Phi \\
-\sin \phi_{1} \sin \phi_{2}+\cos \phi_{1} \cos \phi_{2} \cos \Phi & \cos \phi_{2}-\sin \Phi \\
-\cos \phi_{1} \sin \Phi & \cos \Phi
\end{array}\right)
$$

In the Euler space three subspaces exist in the given range, as seen in Figure 1 without specific sample symmetry, each of which contains every possible orientation once and only once.

Alternatively the rotation matrix $\mathrm{g}$ can be expressed by using the 6 Miller indices of a reference plane, $(h k l)$, and of a reference direction, [uvw], in this plane. For instance the indices of the sheet plane and the rolling direction are commonly used for the description of texture in sheet metals of cubic crystal

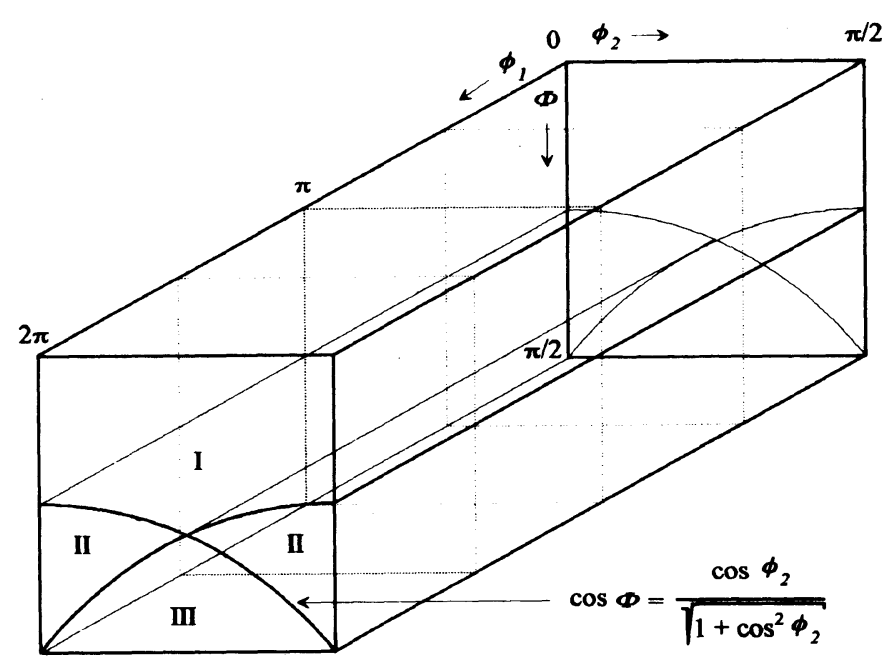

Figure 1 Euler space for cubic crystal and triclinic sample symmetry. 
symmetry (Hansen, 1978; Bunge, 1982):

$$
\mathbf{g}((h k l)[u v w])=\left(\begin{array}{ccc}
\frac{u}{n} & \frac{k w-l v}{m n} & \frac{h}{m} \\
\frac{v}{n} & \frac{l u-h w}{m n} & \frac{k}{m} \\
\frac{w}{n} & \frac{h v-k u}{m n} & \frac{l}{m}
\end{array}\right)
$$

with normalization according to:

$$
m=\sqrt{\left(h^{2}+k^{2}+l^{2}\right)} \quad n=\sqrt{\left(u^{2}+v^{2}+w^{2}\right)}
$$

Finally a single rotation axis, specified by its normalized Miller indices, $\left[t_{x} t_{y} t_{z}\right]$, and an angle of rotation, $\omega$, may be used for describing an orientation. The rotation matrix $g\left(\left(t_{x} t_{y} t_{z}\right), \omega\right)$ has the following form (Hansen, 1978):

$$
\mathbf{g}\left(\left(t_{x} t_{y} t_{z}\right), \omega\right)=\left(\begin{array}{cc}
\left(1-t_{x}^{2}\right) \cos \omega+t_{x}^{2} & t_{x} t_{y}(1-\cos \omega)+t_{z} \sin \omega \\
t_{x} t_{y}(1-\cos \omega)-t_{z} \sin \omega & \left(1-t_{y}^{2}\right) \cos \omega+t_{y}^{2} \\
t_{x} t_{z}(1-\cos \omega)-t_{y} \sin \omega & t_{y} t_{z}(1-\cos \omega)+t_{x} \sin \omega \\
t_{x} t_{z}(1-\cos \omega)+t_{y} \sin \omega \\
t_{y} t_{z}(1-\cos \omega)+t_{x} \sin \omega \\
\left(1-t_{z}^{2}\right) \cos \omega+t_{z}^{2}
\end{array}\right)
$$

A Rodrigues vector, $\mathbf{r}$, can be defined (Frank, 1988) from a rotation axis/angle pair by selection of the smallest possible rotation angle, $\omega$, and normalization of

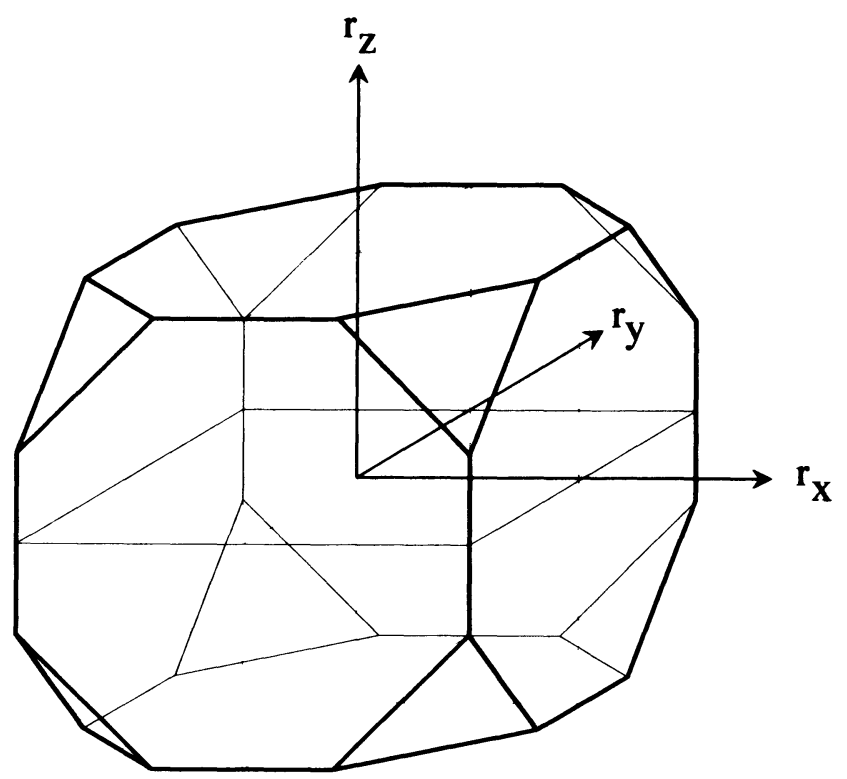

Figure 2 Rodrigues space for cubic crystal and triclinic sample symmetry. 
the vector $t$ of the rotation axis according to:

$$
\mathbf{r}=\mathbf{t} \cdot \tan \frac{\omega}{2}
$$

In all cases of crystal symmetry the smallest rotation angle is less than $62.8^{\circ}$. The basic Rodrigues vectors define a three-dimensional Rodrigues space. In Figure 2 the Rodrigues space without specific sample symmetry is given. The shortest vectors reaching the surface are found along the axes at $\tan \left(45^{\circ} / 2\right)$. The shortest radius vector, along the $\langle 111\rangle$ directions reaching the truncating triangles has a length of $\tan \left(60^{\circ} / 2\right)$. The maximum angle is found at the corners $\tan \left(62.8^{\circ} / 2\right)$. The rectangular coordinates of the corner are $(\sqrt{2}-1, \sqrt{2}-1,3-\sqrt{8})$ (Neumann, 1991). If the sample has a specific symmetry, the orientation cell is that section of the truncated cube which is cut out by the orientation triangle of the sample symmetry (Neumann, 1991).

\section{Orientation Images}

For a graphical representation of grain orientations, the three orientation parameters may be assigned to three basic colours that are red, green and blue. Each grain in a micrograph may thus be stained by a unique colour which, on the other hand, can be interpreted in terms of orientation parameters by comparison with a colour legend. Finally an orientation image of the sample is obtained which illustrates the morphology of microstructure and the orientation distribution of the grains. A computer program was developed for the construction of an orientation image using the measured individual grain orientations and a digitized micrograph of the sample region. A comparison was made between the representations using Miller indices, Euler angles or Rodrigues vectors for orientation parametrisation. In all kinds of representation, however, specific disadvantages become apparent. The best representation depends on the given texture and on the physical problem which is considered.

For orientation images using Miller indices, the three corners of the stereographic standard triangle (inverse pole figure) correspond to the basic colours, i.e. the colour triangle is overlayed on the stereographic standard triangle (Inokuti et al., 1987). For visual clarity, however it is convenient to paint the center in dark (rather than white as in the standard colour triangle) and the corners in bright by the following intensity normalization. The proportion of the intensities of the three basic colours $I_{R}, I_{G}, I_{B}$ is:

$$
\begin{gathered}
I_{R}: I_{G}: I_{B}=\frac{r}{\sqrt{2}-1}: \frac{\beta}{45^{\circ}}\left(1-\frac{r}{\sqrt{2}-1}\right):\left(1-\frac{\beta}{45^{\circ}}\right)\left(1-\frac{r}{\sqrt{2}-1}\right) \\
r=\sqrt{\left(\frac{m}{o+1}\right)^{2}+\left(\frac{n}{o+1}+1\right)^{2}} \beta=\arctan \left(\frac{m}{n}\right)
\end{gathered}
$$

$m, n, o$ are the normalized Miller indices of the considered direction.

In the case of parametrisation by Euler angles the three angular parameters $\phi_{1}$, $\Phi, \phi_{2}$ are assigned to the intensities of the three colours according to:

$$
I_{R}: I_{G}: I_{B}=\frac{\phi_{2}}{90^{\circ}}: \frac{\Phi-55^{\circ}}{35^{\circ}}: \frac{\phi_{1}}{360^{\circ}}
$$


Since subspace III of Figure 1 is less distorted than subspaces I and II (van Houtte, 1987), subspace III is used in the following for a transformation of the Euler angles into colours. In the other subspaces, the same colours from subspace III were taken for each equivalent point of orientation.

For orientation images using the Rodrigues space (Dingley et al. 1991) the values of the three components of a Rodrigues vector, $r_{x}, r_{y}, r_{z}$, are transformed into corresponding intensities of the three basic colours according to:

$$
I_{R}: I_{G}: I_{B}=\frac{r_{y}+\sqrt{2}-1}{\sqrt{2}-1}: \frac{r_{x}+\sqrt{2}-1}{\sqrt{2}-1}: \frac{r_{z}+\sqrt{2}-1}{\sqrt{2}-1}
$$

\section{Comparison of Orientation Representations}

In the following advantages and disadvantages of orientation images will be discussed in more detail. A straightforward graphical representation of crystallographic directions is obtained by using Miller indices. In thin aluminium films on silicon substrates the layer normal is a direction physically preferred by the deposition process as well as by the build-up of stress and strain during heating. Many sputtered or evaporated films are composed of columnar grains which grow in a parallel direction with the layer normal. For a physical interpretation of local texture it is adequate to depict the Miller indices of this reference direction with respect to each grain in an inverse pole figure. Inverse pole figures, however, suffer from a pronounced distortion of the orientation space. The ratio between an area in a.distortion-free ("homochoric") orientation space (Frank, 1988) and in the inverse pole figure is $61 \%$ in the standard triangle 001-011-111 (van Houtte, 1987). Furthermore, two inverse pole figures are necessary for the representation of crystal orientations, e.g. one for the Miller indices of the sample surface plane and another one for an arbitrary reference direction in this plane. Hence it is necessary to present two orientation images of the layer for a full description of crystal texture. In each image grains with a direction closely in common (normal or in the layer plane) are represented by similar colours.
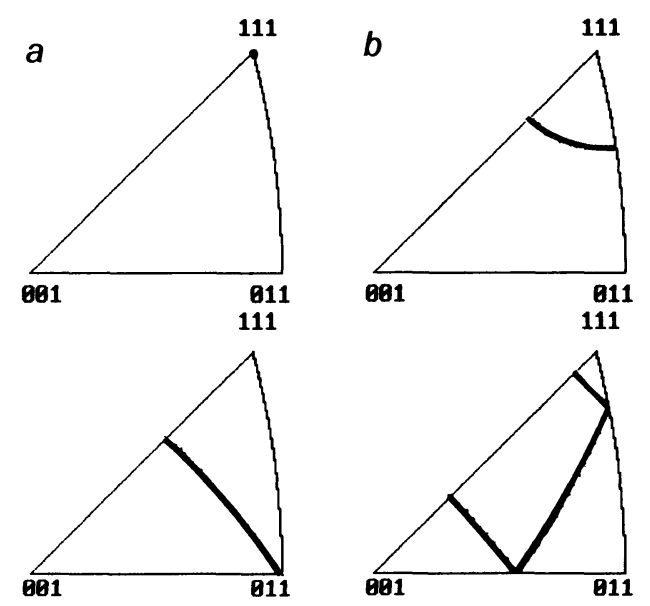

Figure 3 Representation of simulated $\langle 111\rangle$ fibre textures by Miller indices showing inverse pole figures. a) fibre axis parallel with layer normal; b) fibre axis tilted by $15^{\circ}$ to layer normal. 
The full grain orientation may be reconstructed from the two colour images in the following way. From the symmetrically equivalent orientations only those are used for imaging whose Miller indices of the sample normal direction $[h k l]$ fall in the standard triangle 001-011-111. One of the permutations of the Miller indices $\langle u v w\rangle$ of an orthogonal direction is given in the standard triangle 001-011-111. For a full description of crystal orientation the sign and sequence of the Miller indices $[u v w]$ is determined considering the condition of orthogonality between $[h k l]$ and $[u v w]$. If this condition is fulfilled for more than one of the permutations of $\langle u v w\rangle$, these solutions are symmetrically equivalent. An example for the representation by Miller indices is given in Figure 3 by simulated fibre textures $(\langle 111\rangle$ fibre axis). One fibre axis lies in the normal direction of the sample (Figure $3 a$, left), the other is tilted by $15^{\circ}$ around the $y$-axis of the sample (Figure 3b, right).

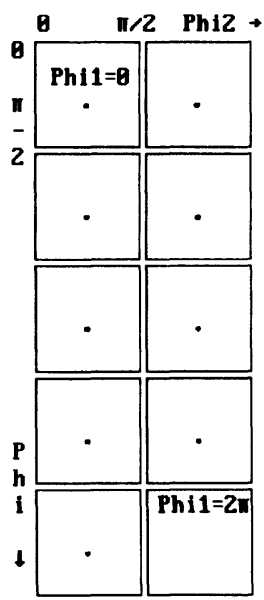

$a$
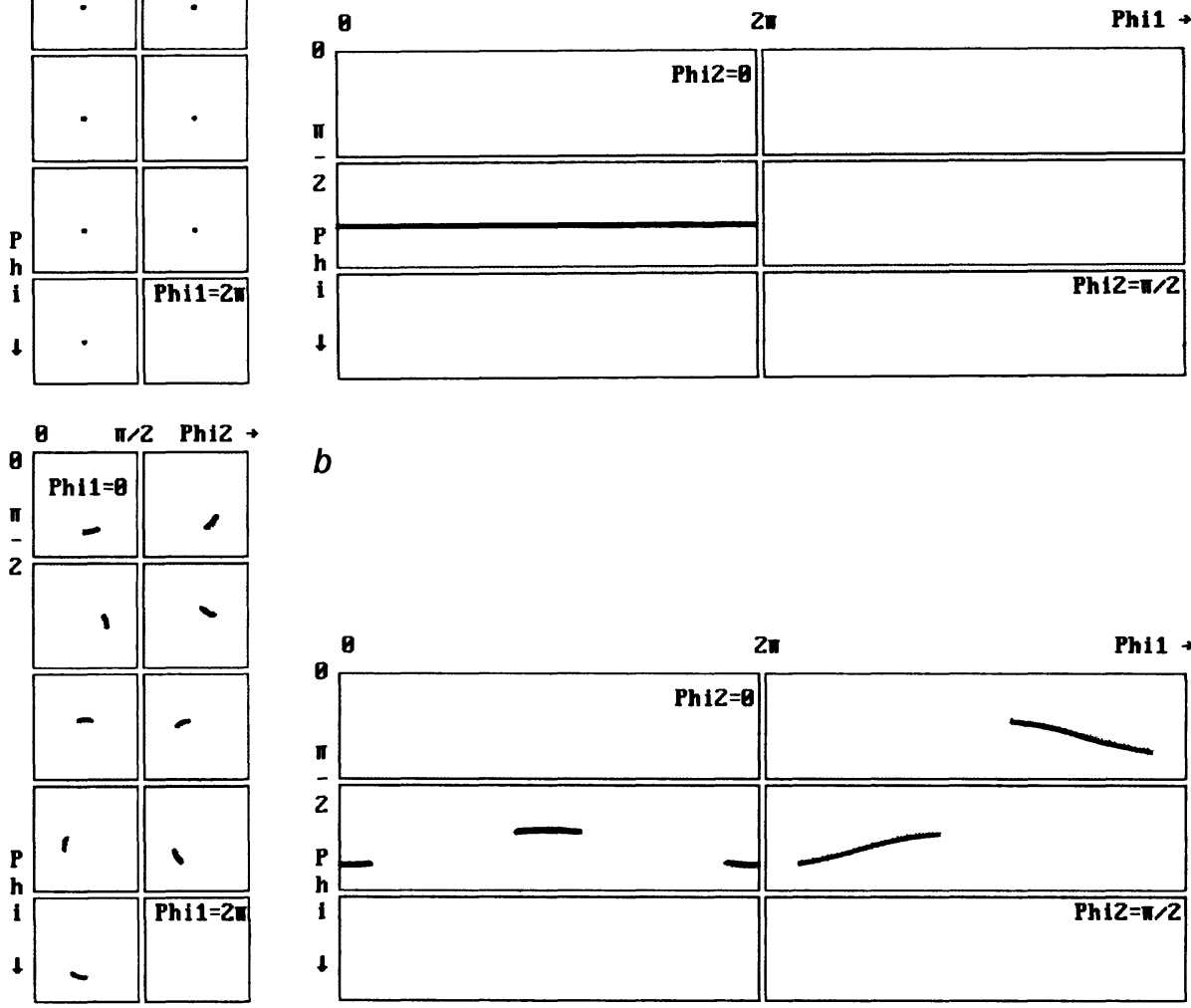

b

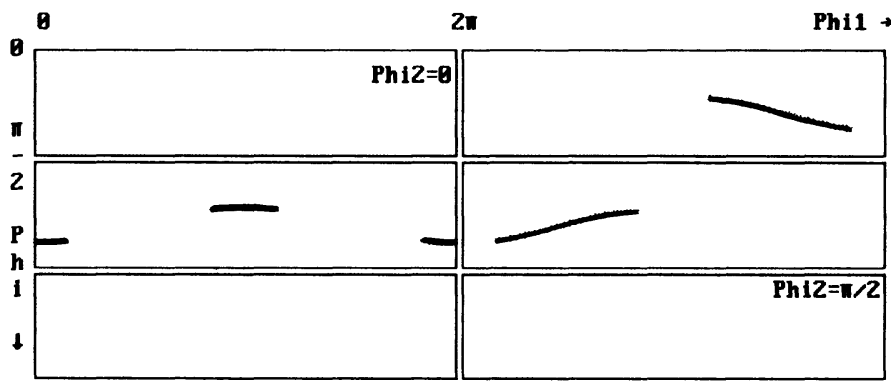

Figure 4 Representation of simulated (111) fibre textures by Euler angles (Symmetry cubictriclinic). The orientation points are projected to their nearest section plane. a) fibre axis parallel with layer normal; $b$ ) fibre axis tilted by $15^{\circ}$ to layer normal. 
In the Euler space each orientation is present three times, and the orientation space is distorted. The ratio between an area in a distortion-free orientation space and an area in the Euler space is here less than 22.5\% (van Houtte, 1987). A fibre texture with the fibre axis in the normal direction of the sample, as found for example in the case of thin sputtered aluminium films $(\langle 111\rangle$ fibre axis), is represented by a straight line, since $\Phi$ and $\phi_{2}$ are constant and only $\phi_{1}$ will change, or $\phi_{2}$ is constant and $\phi_{1}=0$ and only $\Phi$ will change. A fibre texture with a fibre axis in an oblique direction to the sample normal, however, is given by a curved line in Euler space. This is demonstrated by Figure 4 for the same simulated $\langle 111\rangle$ fibre textures as in Figure 3. The usual representations by $\phi_{2}=$ constant sections and $\phi_{1}=$ constant sections are given. The $\phi_{1}=$ constant section representation with restriction to subspace III as proposed by (van Houtte, 1987) was used, due to the smallest distortion of the orientation space and the close relation between Euler space and inverse pole figures. Subspace III (cf. Figure 1) is related to the standard triangles 110-110-111 and 010-110-111 (van Houtte, 1987). The inverse pole figure of the normal direction of the sample is obtained by projection of the orientation distribution along the $\phi_{1}=$ constant sections, and a fibre texture with the fibre axis in the sample normal direction (Figure $4 \mathrm{a}$, top) is represented by points in the $\phi_{1}=$ constant sections in Euler space. If the fibre axis is tilted with respect to the layer normal, the individual grain orientations lie on curved lines in the Euler space (Figure 4b). The visual interpretation of orientations in an orientation image, is not easily done due to the difficulty. in extracting the intensities of the basic colours from a mixed colour. Similar orientations, however, are represented by similar colours.

a

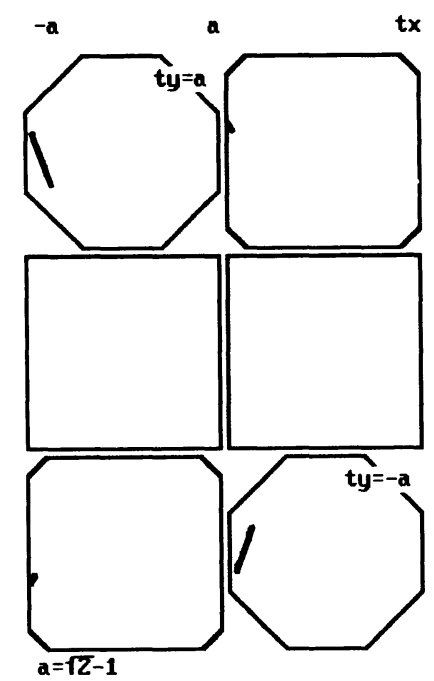

b

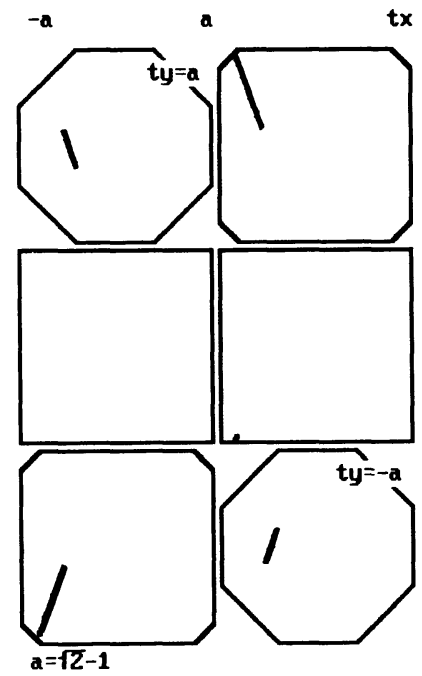

Figure 5 Representation of simulated $\langle 111\rangle$ fibre textures by Rodrigues vectors (Symmetry cubic-triclinic). The orientation points are projected to their nearest section plane. a) fibre axis parallel with layer normal; $b$ ) fibre axis tilted by $15^{\circ}$ to layer normal. 
For comparison, the same fibre textures as in Figures 3 and 4 are given in Figure 5 using Rodrigues vectors. The bounding surfaces of the Rodrigues space are planes in all cases of specific symmetry (Frank, 1988; Neumann, 1991). The Rodrigues space is distorted as well. The invariant volume element, however, deviates from the volume element in a distortion-free orientation space by less than a factor of two (Neumann, 1991). The indices of the rotation axis may be negative, so the origins of colour and Rodrigues space are not congruous. In the consequence same rotation axes but different rotation angles, i.e. collinear Rodrigues vectors but different absolute values, are not represented by colours with similar intensities of the basic colours (see also Eq. (13)). All fibre textures are represented by a straight line in Rodrigues space, irrespective of the angle with respect to the layer normal as a reference direction (Figure 5a and 5b). By selecting the smallest rotation angle, however, one may jump from one rotation axis to another. The result is that grains with a similar orientation may then fall on rather different colours ("umklapp effect").

\section{EXPERIMENTAL}

Investigations were carried out with $800 \mathrm{~nm} \mathrm{Al}-1 \%$ Si layers on thermally oxidized (001) silicon substrates. The thickness of the silicon oxide layer was about $100 \mathrm{~nm}$. The films were sputter deposited at room temperature (RT) at a deposition rate of $10 \mathrm{~nm} / \mathrm{s}$.

The samples were annealed under vacuum. Two different heat treatments were applied:

- direct heating to a fixed temperature $\left(400^{\circ} \mathrm{C}\right.$ or $\left.550^{\circ} \mathrm{C}\right)$ with a further anneal for 30 minutes (called isothermal annealing) and

-stepwise heating from RT up to $550^{\circ} \mathrm{C}$ in steps of $50^{\circ} \mathrm{C}$ and 6 minutes annealing time.

For transmission electron microscopy, samples were prepared by chemical polishing. The back side of a sample was thinned to a hole by dipping the disk in a mixture of $\mathrm{HNO}_{3}$ and $\mathrm{HF}(6.5: 1)$. The aluminium layer was protected from etching by a droplet of glycerine under a plastic lid.

The morphology and the individual crystal orientations of the grains after annealing were investigated in a TEM at an accelerating voltage of $300 \mathrm{kV}$ (PHILIPS EM 430). The crystal orientations were measured on-line using microbeam Kikuchi patterns (Schwarzer, 1990). For each type of sample the orientations of all grains in a selected region (about 200 matrix grains and a few hillocks) were determined. Hillock density, however is low and consequently the number of hillocks found in a thus selected region is not sufficient for meaningful statistics. Hence the orientations of about 20 more hillocks and of their surrounding grains in similar regions were measured in addition. The micrographs of the areas with the measured grains were digitized. From the measured single grain orientations the data of the orientation parametrisations are calculated by a computer program. The results are represented by orientation images of the grain orientations. 


\section{RESULTS AND DISCUSSION}

Figure 6 shows the grain structure of the annealed samples by electron micrographs. All the films have a columnar structure after annealing at about $200^{\circ} \mathrm{C}$ (Gerth et al., 1992b). Grain growth occurs in the temperature range from about $100^{\circ} \mathrm{C}$ to the final temperature. Some hillocks are growing during the anneal process. The micrograph of a stepwise heated sample (Figure $6 \mathrm{~d}$ ) contains three hillocks which form dark patches. In the other samples (Figure 6a-6c) hillocks were found, too, but they are not in the range of the micrographs of Figure 6.

The orientation distributions of the grains in the $\mathrm{Al}-1 \% \mathrm{Si}$ layers are given in Figure 7 by inverse pole figures for the plane normal direction and for an arbitrary reference direction in the layer plane. It turns out that the majority of the grains are closely oriented along a $\langle 111\rangle$ fibre texture. There are no preferred crystal directions present in the layer plane. This texture is represented by straight lines in the Rodrigues space (Figure 8 ) as well as in Euler space along $\phi_{2}=45^{\circ}$ and $\Phi=5.73^{\circ}(=\arccos (1 / \sqrt{3})$ (Figure 9$)$. On the as-deposited samples a $\langle 111\rangle$ fibre texture was confirmed by $x$-ray pole figure measurement. Strength of texture is reduced during grain growth at high temperatures. Texture is sharper after stepwise heating up to $550^{\circ} \mathrm{C}$ than after isothermal heating at $550^{\circ} \mathrm{C}$.

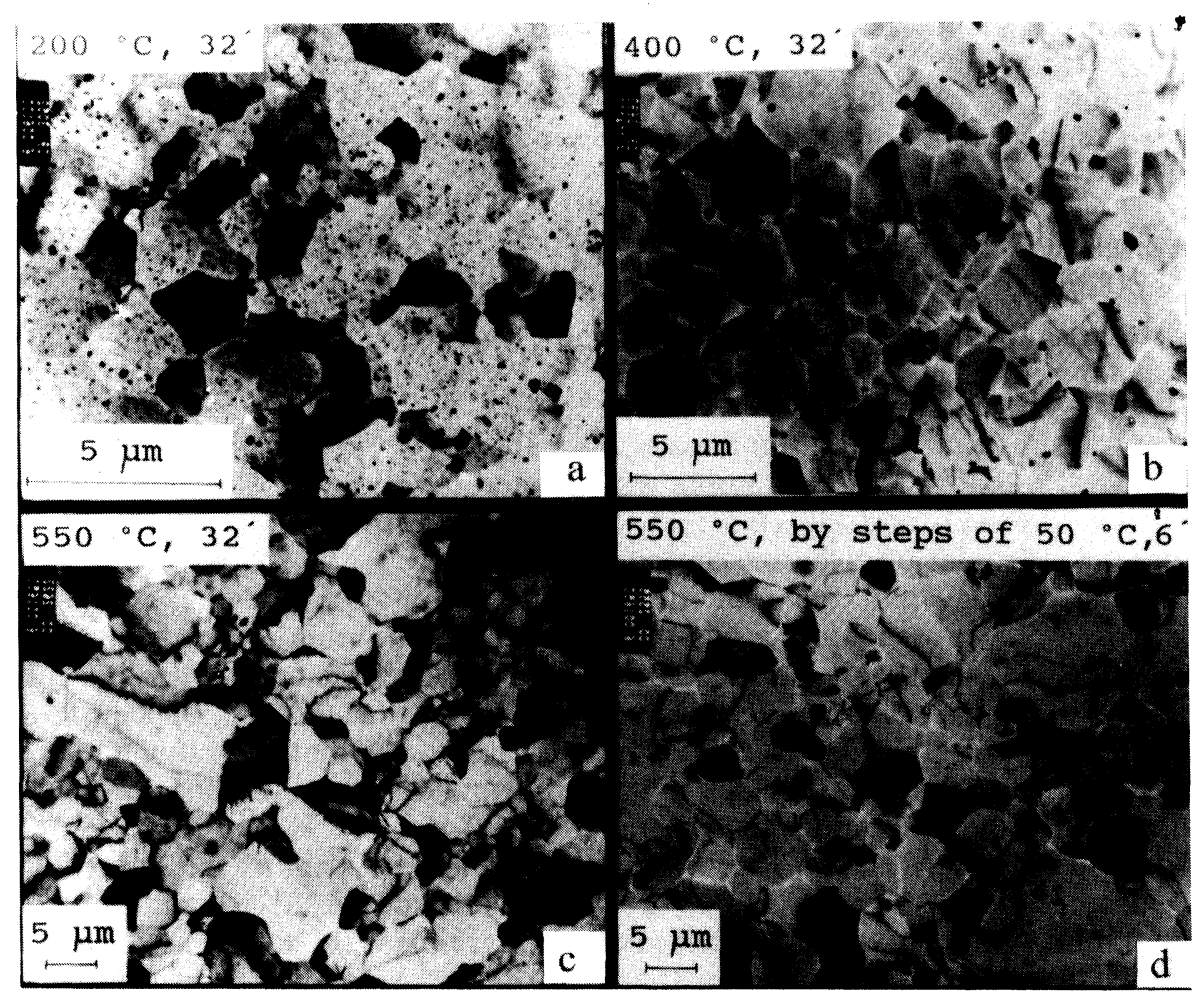

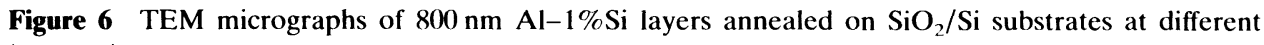
temperatures. 


\section{Normal direction}
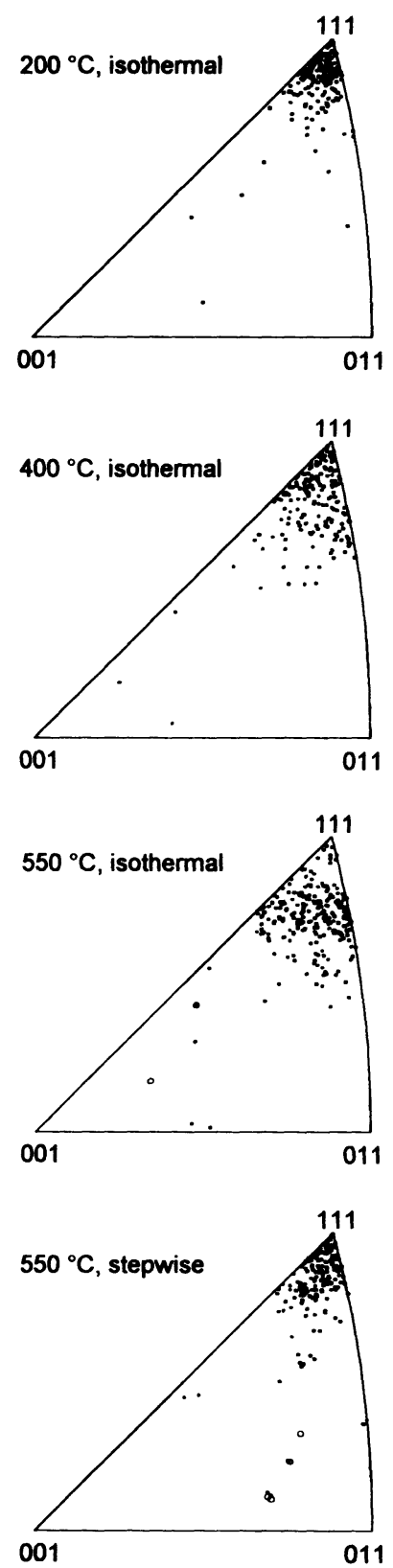

Azimuthal direction
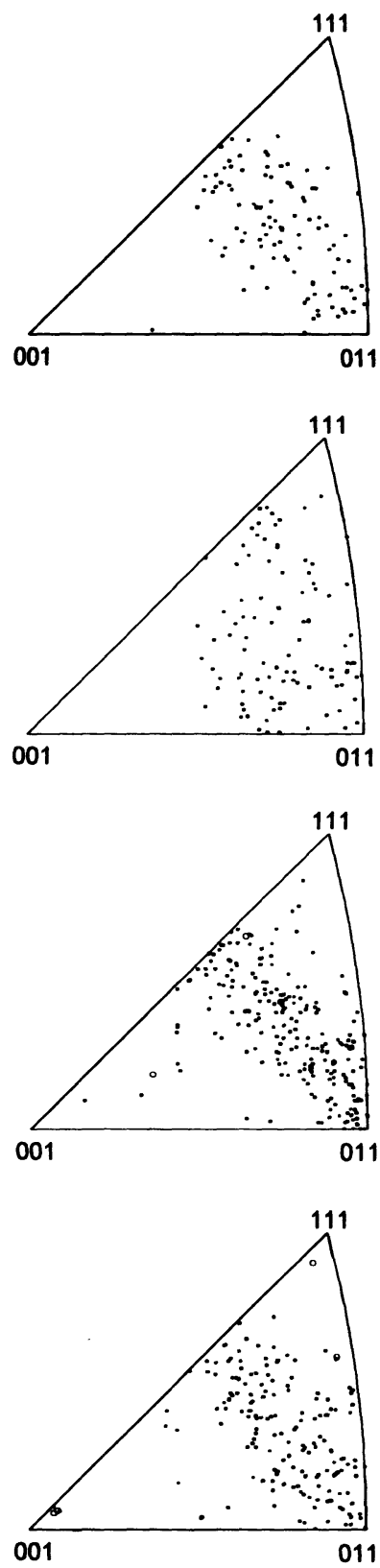

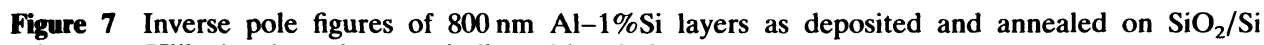
substrates. Hillock orientations are indicated by circles. 

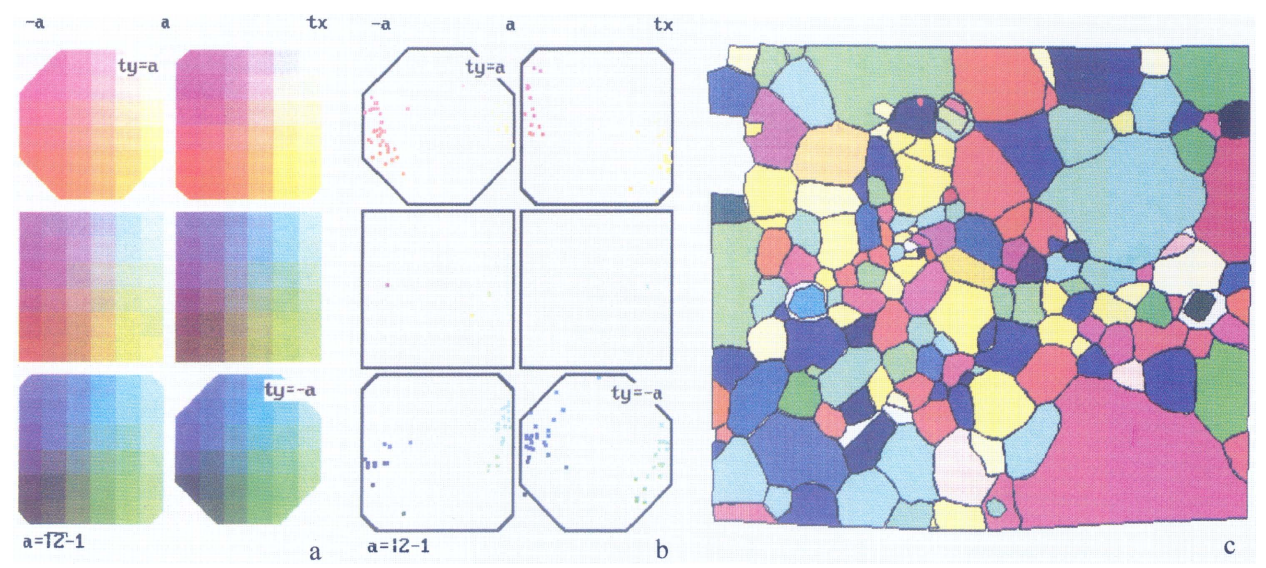

Figure 8 (see Colour Plate at the back of this issue) Orientation image using Rodrigues vectors. Sample: $800 \mathrm{~nm} \mathrm{Al-1 \% Si} \mathrm{layer} \mathrm{on} \mathrm{Si} \mathrm{substrate} \mathrm{after} \mathrm{stepwise} \mathrm{annealing} \mathrm{from} \mathrm{RT} \mathrm{to} 550^{\circ} \mathrm{C}$ (Symmetry cubic-triclinic). a) colour key; b) individual orientations in Rodrigues space; c) orientation image.

The orientation distribution of grains adjacent to hillocks is given in Figure 10 by inverse pole figures for the plane normal direction and the arbitrary reference direction in the layer plane. Many grains deviate from the pronounced $\langle 111\rangle$ fibre texture of the layer. In the stepwise heated sample the grains in the neighbourhood of hillocks have a sharper $\langle 111\rangle$ fibre texture than in the isothermally annealed one. After annealing up to $400^{\circ} \mathrm{C}$ the hillocks have the same orientations as the underlying grain, but after annealing up to $550^{\circ} \mathrm{C}$ some hillocks differ in orientation, as a result of grain growth and hillock recrystallization.

The conclusion is drawn that the hillocks start growing in regions with a local texture deviating from the ordinary $\langle 111\rangle$ fibre texture of the layer. In the beginning the hillocks have the same orientations as the grains beneath. Also polycrystalline hillocks were found. Other investigations (Ericson et al., 1991; Gerth et al., 1992b) support the conclusion that hillocks grow above grain boundaries or triple point junctions. During further heating, the grains beneath may coalesce with neighbouring grains having a $\langle 111\rangle$ orientation while the hillocks retain their initial orientation. Therefore at higher temperatures, hillocks may have an orientation quite different from the underlying grains. Hillocks even above a single grain were found. In these cases grain growth of the underlying grains may have annihilated the grain boundary or the triple point junction beneath the hillock (Gerth et al., 1992a). Hillock recrystallization may also occur (Ericson et al., 1991), and as a result some hillocks with a $\langle 111\rangle$ orientation are formed, particularly at higher temperatures.

As an example the different textures of hillocks and grains are shown in orientation images of the stepwise heated layer in terms of Rodrigues vectors, Euler angles and Miller indices (Figures 8, 9 and 11). The hillock orientations differ from the grain orientations of the layer which is illustrated by colour differences. The clearest plot of this finding is given by Miller indices repre- 

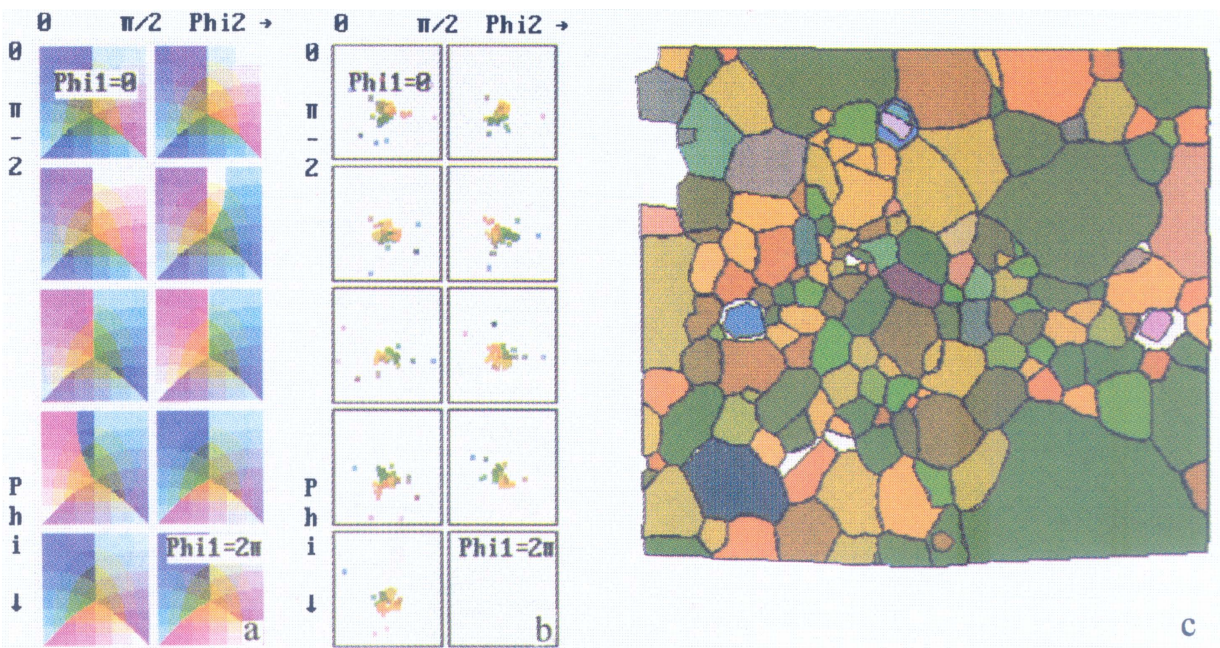

c
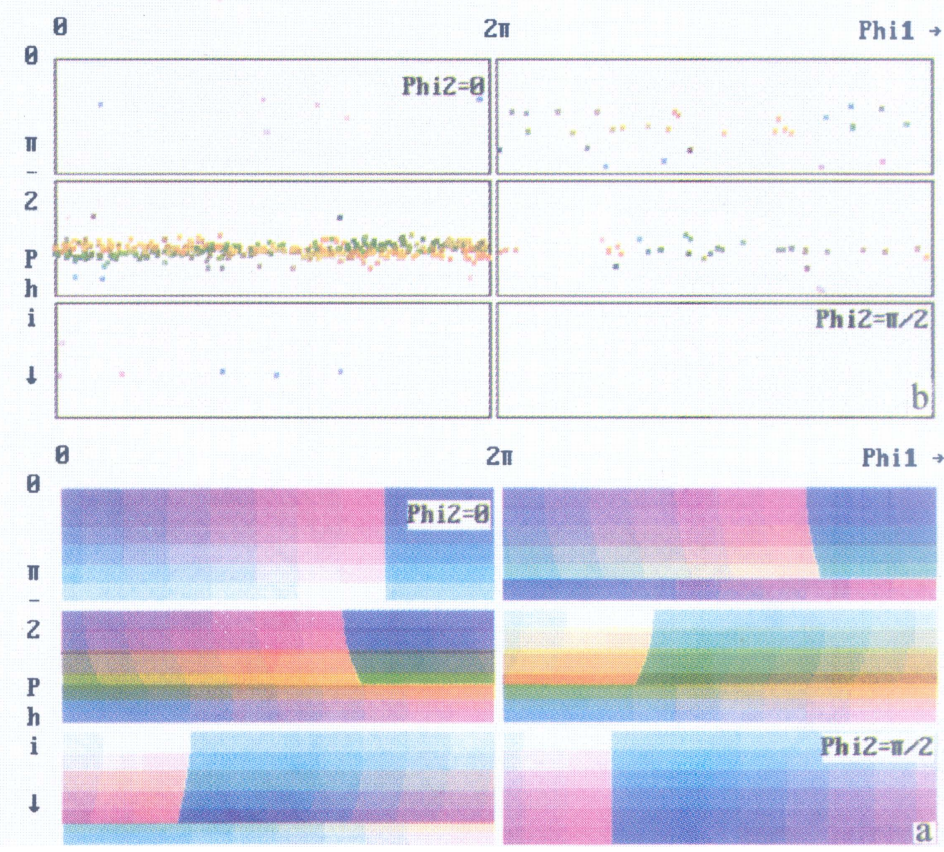

Figure 9 (see Colour Plate at the back of this issue) Orientation image using Euler angles. (Sample as Figure 8). a) colour key; b) individual orientations in Euler space; c) orientation image. 

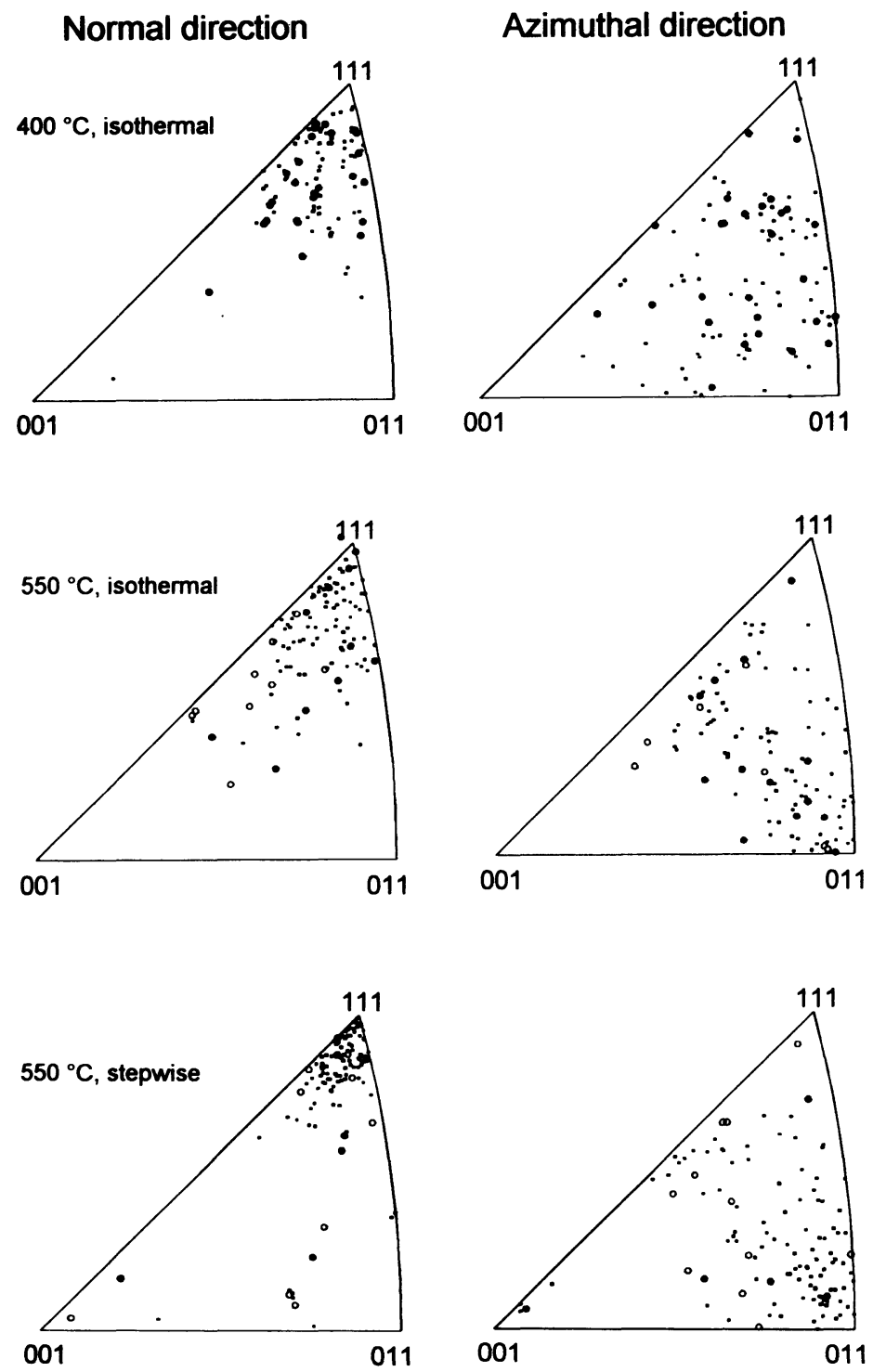

Figure 10 Inverse pole figures of grains neighbouring hillocks. Samples: $800 \mathrm{~nm} \mathrm{Al-1 \% Si} \mathrm{layers}$ annealed on $\mathrm{SiO}_{2} / \mathrm{Si}$ substrates at different temperatures. Hillock orientations are indicated by circles. 


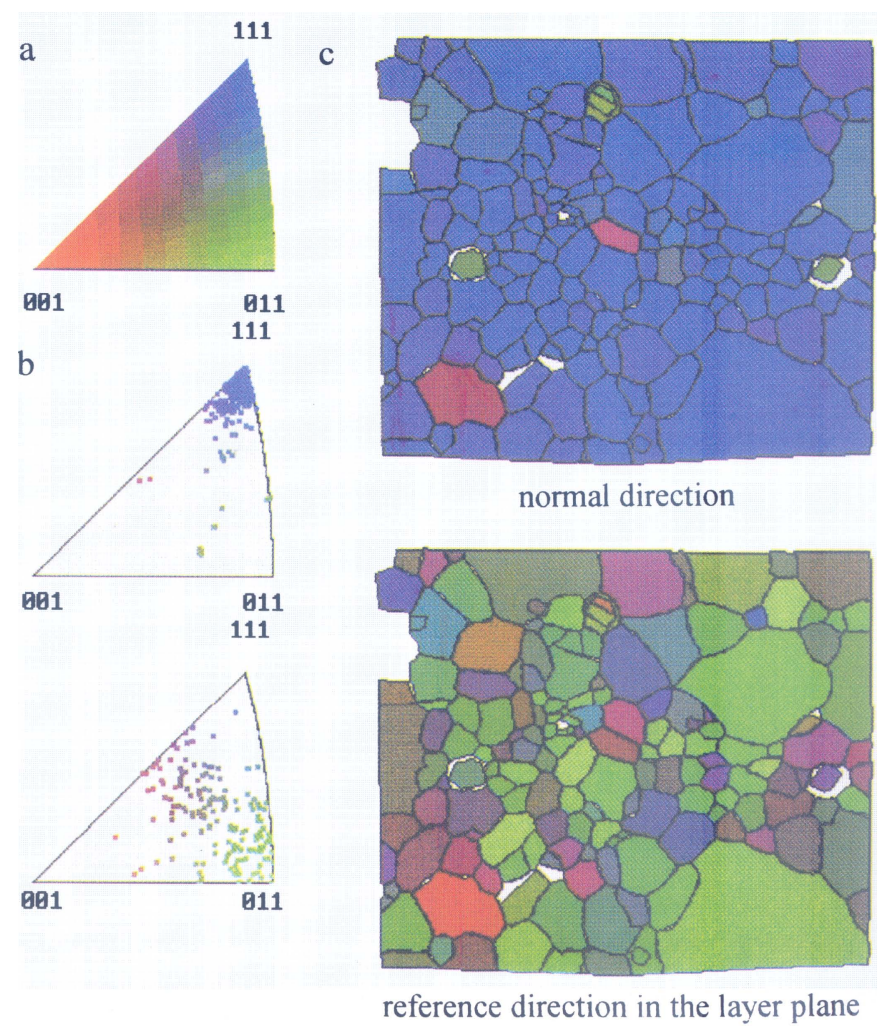

Figure 11 (see Colour Plate at the back of this issue) Orientation image using Miller indices and inverse pole figures (top. normal direction, bottom: reference direction in the layer plane). (Sample as Figure 8). a) colour key; b) individual orientations; c) orientation images.

sentation: While all matrix grains appear in nearly the same (blue) colour in the normal direction image, the hillocks contrast from the preferred $\langle 111\rangle$ fibre axis of the matrix by striking colours.

\section{CONCLUSION}

The measurement of crystal orientations of single grains and hillocks in annealed $\mathrm{Al}-1 \% \mathrm{Si}$ layers on oxidized silicon substrates reveals a deviation of the local textures of hillocks and their surrounding grains from the ordinary $\langle 111\rangle$ fibre texture of the layers. A grain-specific distribution of orientations is clearly displayed by orientation images using Miller indices, Euler angles or Rodrigues vectors. In the case of a $\langle 111\rangle$ fibre texture, as in sputter-deposited thin aluminium films on a silicon substrate, the most appropriate orientation image is obtained by using Miller indices for orientation parametrisation with one reference direction coincident with the fibre axis. 
Hillocks are concluded to start growing on points with an orientation deviating from the $\langle 111\rangle$ fibre texture of the layer. After heat treatments at higher temperatures the crystal orientations of the hillocks and the underlying grains may change by grain growth and recrystallization processes.

\section{ACKNOWLEDGEMENT}

The authors would like to thank S. Zaefferer for many helpful discussions. This work was made possible by an award from the German Ministry of Science and Technology (BMFT). Financial support from the German Research Foundation (DFG project Bu 374/22) is gratefully acknowledged.

\section{References}

Bacconier, B., Lormand, G., Papapietro, M., Achard, M. and Papon, A.-M. (1988). A study of heating rate and texture influences on annealing hillocks by a statistical characterization of $\mathrm{Al}$ thin-film topography. Journal of Applied Physics, 64, 6483-6489.

Bunge, H. J. (1982). Texture Analysis in Materials Science. Butterworths London 1982.

Chang, C. Y., Vook, R. W., Lee, Y. C. and Hoshi, I. (1989). Isothermal annealing of hillocks in Al-Cu films. Thin Solid Films 181, 57-63.

Chaudhari, P. (1974). Hillock growth in thin films. Journal of Applied Physics 45, 4339-4346.

Dingley, D. J., Day, A. and Bewick, A. (1991). Application of microtexture determination using EBSD to non cubic crystals. Textures and Microstructures 14-18, 91-96.

Ericson, F., Kristensen, N., Schweitz, J.-A. and Smith, U. (1991). A transmission electron microscopy study of hillocks in thin aluminum films. Journal of Vacuum Science and Technology B9, 58-63.

Frank, F. C. (1988). Orientation mapping. Metallurgical Transactions 19A, 403-408.

Gerth, D., Katzer, D. and Schwarzer, R. A. (1992a). Correlation between grain growth and hillock growth in thin thermally annealed $\mathrm{Al}-1 \% \mathrm{Si}$ films on silicon substrates. Material Science Forum 94-96, 556-561.

Gerth, D., Katzer, D. and Krohn, M. (1992b). Study of the thermal behaviour of thin Al alloy films. Thin Solid Films 208, 67-75.

Hansen, J., Pospiech, J. and Lücke, K. (1978). Tables for Texture Analysis of Cubic Crystals. Springer-Verlag Berlin-Heidelberg-New York 1978.

d'Heurle, F. M. (1989). Metallurgical topics in silicon device interconnections: Thin film stresses. International Materials Review 34, 53-68.

van Houtte, P. (1987). On the representation of texture functions of cubic metals in Euler space. Textures and Microstructures 7, 187-205.

Inokuti, Y., Maeda, C. and Ito, Y. (1987). Computer colour mapping of configuration of Goss grains after transverse cold rolling in grain oriented silicon steel. Transactions of the Iron Steel Institute of Japan 27, 302-311.

Jackson, M. S. and Li, C.-Y. (1982). Stress relaxation and hillock growth in thin films. Acta Metallurgica 30, 1993-2000.

Kim, J. Y. and Hummel, R. E. (1990). Hole and hillock formation in gold metallizations. Physica Status Solidi 122, 255-273.

Lahiri, S. K. (1970). Stress relief and hillock formation in thin lead films. Journal of Applied Physics, 41, 3172-3176.

Neumann, N. (1991). Graphical representations of orientations and ODFs by Rodrigues vectors. Steel Research 62, 560-566.

Pennebaker, W. P. (1969). Hillock growth and stress relief in sputtered Au films. Journal of Applied Physics, 40, 394-400.

Sanchez, J. E. Jr. and Arzt, E. (1992). Effects of grain orientation on hillock formation and grain growth in aluminum films on silicon substrates. Scripta Metallurgica 27, 285-290. 
Schwarzer, R. A. (1990). Measurement of local textures with transmission and scanning electron microscopes. Textures and Microstructures 13, 15-30.

Smith, U., Kristensen, N., Ericson, F. and Schweitz, J.-Å. (1991). Local stress relaxation phenomena in thin aluminum films. Journal of Vacuum Science and Technology A9, 2527-2535.

Zimmermann, Z. (1988). Einsatz eines heizbaren Probentisches im Rasterelektronenmikroskop zur Untersuchung von $\mathrm{Al} / \mathrm{Si}-1 \%$ Metallisierungsschichten in der Mikroelektronik. Beiträge zur elektronenmikroskopischen Direktabbildung von Oberfächen 21, 159-170. 